Please cite this paper as follows:

Hong-Ki Hong and Jeng-Tzong Chen, Derivations of Integral Equations of Elasticity, ASCE Journal of Engineering Mechanics, Vol.114, No.6, pp.1028-1044, 1988. 


\title{
Derivations of Integral Equations of Elasticity
}

\author{
By Hong-Ki Hong1 and Jeng-Tzong Chen ${ }^{2}$
}

\begin{abstract}
In this paper, we derive the integral equations of elasticity, which may be considered to be a very general formulation for solutions of (cracked and uncracked) elasticity problems. The formulation is general enough to be a starting point for an analytical study or for a numerical treatment. The theory can be developed either by utilizing Betti's law or the weighted residual method, or directly resorting to physical meaning, as in the potential theory. To show that the results of the derivations are consistent with one another, we also prove four lemmas of the properties of the kernel functions. The derivations are continued by applying two commutative operations, traction and trace, leading naturally to the concept of Hadamard principal value. Consequently, singularity, often present in problems involving geometry degeneracy, causes no particular difficulties. Finally, we employ two examples to demonstrate the usefulness of the resulting dual boundary integral equations in both analytical and numerical solution procedures.
\end{abstract}

\section{INTRODUCTION}

Interest in the mathematical theory of integral equations developed in the years following 1900 after the Swedish geometer E. I. Fredholm developed his remarkable theory. The subject flourished for some 40 years. This was followed by decreasing activity, which lasted about 30 years, until the advent of electronic digital computers provided a stimulus to a renewed interest in the subject (Elliott 1980). Today, under the headings of functional analysis, numerical analysis, and finite element, approximation theory further promotes the study of integral equations; in particular, the so-called boundary-element method (BEM) has been drawing much attention. As a prelude to a modern BEM treatment of elasticity problems, Rizzo (1967) presented his formulation of the so-called "direct method" of linear elasticity based on Somigliana's identity. The boundaryintegral equation obtained represents a compatible relation between the traction and displacement on the boundary. To solve the integral equation, Rizzo was able to obtain a system of algebraic equations for the boundary unknowns upon employing a systematic discretization technique. The traction and displacement fields can thus be calculated from the integral equation after the solutions for all the boundary unknowns have been obtained. On the other hand, even earlier, in 1965, a seemingly different approach, the so-called "indirect method," in essence first presented by Lauricella (1909) (Muskhelishvili 1953), was put forth by Massonnet (1965) and Kupradze (1965). In this approach, the region of interest is embedded in the infinite domain, which has the same material property as the original and the material of which must be homogeneous throughout, and in which

Prof., Dept. of Civ. Engrg., Nat. Taiwan Univ., Taipei 10764, Taiwan.

${ }^{2}$ Grad. Student, Inst. of Appl. Mech., Nat. Taiwan Univ, , Taipei 10764, Taiwan.

Note. Discussion open until November 1, 1988. To extend the closing date one month, a written request must be filed with the ASCE Manager of Journals. The manuscript for this paper was submitted for review and possible publication on November 24, 1986. This paper is part of the Journal of Engineering Mechanics, Vol. 114, No. 6, June, 1988. CASCE, ISSN 0733-9399/88/0006-1028/\$1.00+\$.15 per page. Paper No. 22515. 
Green's functions of a point load source or a dislocation source are obviously much more easily determined.

The direct and indirect methods will be reexamined in this paper; they are shown to be equivalent when investigated under our formulation of dual integral equations. This system also provides a genuinely correct formulation for solving boundary value problems of elastic media with cracks. In this regard, it may be recalled that an insurmountable difficulty in the use of conventional BEM crack modeling stems from a mathematical degeneracy in the BEM formulation when both upper and lower crack surfaces lie in the same location. The equations for the points at the upper surface of the crack are dependent on and even identical to those at the lower, and, as a consequence, the formulation is insufficient to secure a unique solution; indeed, it results in infinitely many solutions. In a numerical calculation, this is evident in a vanishing determinant or algebraic singularity of the coefficient matrix of the discretized governing equations (Cruse 1975).

However, some special techniques have been devised to solve this difficulty. Among others, the special Green's function methods (Cruse 1975) and the zone method (Ligget and Liu 1983) may be worth mentioning. The special Green's function method is applied to problems with a dominant crack of so regular a shape that the free-space Green's function, which satisfies the traction-free boundary condition on the crack surface, is obtainable. The zone method introduces artificial boundaries in the intact area to connect cracks and the boundary and thus divides the domain into zones such that no cracks appear in the interior of each zone. Compatibility and equilibrium are then enforced on the artificial boundaries to restore approximate intactness. The drawback of this latter method is obvious in that the introduction of artificial boundaries is arbitrary, nonunique, and thus not qualified as an automatic scheme. Also, it results in a larger system of equations than needed.

Although the aforementioned special techniques are available, it is clear that there exists in the theory of elasticity a fundamental problem of a lack of a general integral formulation for problems of an elastic body with degenerate geometry that encloses no area or volume. For this, if we start from the other end and examine carefully exact solutions of a handful of crack problems, we may be motivated to realize that a single integral equation of elasticity such as the Somigliana identity is too slim to solve general elastic crack problems. The observation leads us to seek an additional integral equation, finally reaching the so-called dual integral equations. Owing to its generality the dual integral equations may be deemed as a pair of fundamental formulations of the theory of elasticity and linear elastic fracture mechanics.

In the following sections we first derive the Somigliana identity and then apply the traction operator to the identity to yield a second equation. The resulting two equations, being independent, are referred to as the domain dual integral equations, which after the application of a trace operation of extension to the boundary becomes the dual boundary integral equations. We also prove four lemmas of the properties of the kernel functions of the integral equations and the commutativity of the two operations of traction and trace, with which we are thus able to show the consistency of different approaches of derivations. Finally, an analytical example and a numerical 
BEM exercise are given to demonstrate at an elementary level the applicability of the dual integral equations.

\section{Derivations of Dual Domain Integral Equations}

In this section we derive through three different approaches dual integral equations for domain points for general elasticity problems.

\section{Betti's Law}

Let $\left(b_{i}, t_{i}, u_{i}\right)$ and $\left(b_{i}^{*}, t_{i}^{*}, u_{i}^{*}\right)$ be two equilibrium states in a linearly elastic body where $b_{i}, b_{i}^{*}=$ the body forces; $t_{i}, t_{i}^{*}=$ the boundary tractions; and $u_{i}, u_{i}^{*}=$ the displacements. Betti's law of reciprocality gives

$$
\int_{D}\left(u_{i} b_{i}^{*}-u_{i}^{*} b_{i}\right) d V=-\int_{B}\left(u_{i} t_{i}^{*}-u_{i}^{*} t_{i}\right) d B \ldots \ldots \ldots \ldots \ldots
$$

where $D=$ a domain with boundary $B$. It can be recast into the theory of self-adjoint operator $\mathscr{L}$ simply as

$(\mathscr{L} u \mid v)=(u \mid \mathscr{L} v)$

where

$\mathscr{L}=\left[\begin{array}{cc}\mathscr{D} & 0 \\ 0 & -\mathscr{B}\end{array}\right]$

If the linearly elastic material is isotropic, the operator $\mathscr{D}$ is expressed explicitly as

$D_{i j}=(\lambda+G) \partial_{i} \partial_{j}+G \delta_{i j} \partial_{k} \partial_{k}$

while $\mathscr{B}=$ the traction operator defined by

$B_{i j}=\lambda n_{i} \partial_{j}+G\left(n_{j} \partial_{i}+\delta_{i j} n_{k} \partial_{k}\right)$

where $\lambda$ and $G=$ Lame's constants; $n_{i}=$ direction cosines of the unit outward normal to the boundary; $\delta_{i j}=$ Kronecker delta; and $\partial_{i}=$ the partial differential operator. For an anisotropic material, the expressions for $D_{i j}$ and $B_{i j}$ can be found readily, too. Note that the equations of equilibrium, $D_{i j} l_{j}+b_{i}=0$ and $D_{i j} l_{j}^{*}+b_{i}^{*}=0$ in $D$, and the Cauchy formulas, $B_{i j} u_{j}=t_{i}$ and $B_{i j} u_{j}^{*}=t_{i}^{*}$ on $B$, and $u_{j}^{*}=v_{j}$ have been used in arriving at Eq. 2.

To elaborate Eq. 2, we state explicitly that if $D \subset R^{\prime \prime}(n=1,2,3)$ is a regular or otherwise appropriately conditioned domain with adequately conditioned boundary $B$, and if the functions $u_{i}(x)$ and $v_{i}(x)$ are elements of $C^{1}(\bar{D}) \cap C^{2}(D)$ or an appropriate Sobolev space and have a bounded support, then

$$
\int_{D}\left(v_{i} D_{i j} u_{j}-u_{i} D_{i j} v_{j}\right) d V=\int_{B}\left(v_{i} B_{i j} u_{j}-u_{i} B_{i j} v_{j}\right) d B \ldots \ldots \ldots \ldots
$$

If $B$ is unbounded, the condition of bounded support can be replaced by a radiation condition. 
Now choose specifically:

$$
\begin{aligned}
& t_{i}^{*}(x)=B_{i k}(x) v_{k}(x)=B_{i k}(x) U_{k j}(x, s) e_{j}^{*}(s)=T_{i j}(x, s) e_{j}^{*}(s) \ldots \ldots \\
& b_{i}^{*}(x)=-D_{i k}(x) v_{k}(x)=-D_{i k}(x) U_{k j}(x, s) e_{j}^{*}(s)=\delta_{i j}(x, s) e_{j}^{*}(s) \ldots \\
& u_{i}^{*}(x)=v_{i}(x)=U_{i j}(x, s) e_{j}^{*}(s) \ldots \ldots \ldots \ldots \ldots \ldots \ldots
\end{aligned}
$$

where $U_{i j}(x, s)$ and $T_{i j}(x, s)=$ the free-space Green's functions (or fundamental solutions) of displacement and traction, respectively, due to a concentrated load in the $j$-direction at the point $s$; and $e_{j}^{*}(s)=$ an arbitrary unit-concentrated load at the point $s$. Then we have Somigliana's identity (Banerjee and Butterfield 1981): if $b_{i}=0$, then

$$
\begin{aligned}
& \int_{B}\left[U_{i j}(x, s) t_{i}(x)-T_{i j}(x, s) u_{i}(x)\right] d B(x)=u_{j}(s), \text { if } s \in D \ldots \ldots \\
& \int_{B}\left[U_{i j}(x, s) t_{i}(x)-T_{i j}(x, s) u_{i}(x)\right] d B(x)=0, \quad \text { if } s \in D \ldots \ldots
\end{aligned}
$$

which, for later comparison purposes, is changed to

$$
\begin{aligned}
& \int_{B}\left[U_{k i}(s, x) t_{k}(s)-T_{k i}(s, x) u_{k}(s)\right] d B(s)=u_{i}(x), \text { if } x \in D \ldots \ldots \\
& \int_{B}\left[U_{k i}(s, x) t_{k}(s)-T_{k i}(s, x) u_{k}(s)\right] d B(s)=0, \quad \text { if } x \in D \ldots \ldots
\end{aligned}
$$

In deriving Eq. 10 we have omitted the unit vector $e_{j}^{*}$ from both sides of the equation because of its arbitrariness. Now, in order to have an additional, independent equation, we apply the traction operator $B_{p i}$ to Eq. 11 and define

$$
\begin{aligned}
& B_{p i}(x)\left[U_{k i}(s, x)\right]=L_{k p}(s, x) \\
& B_{p i}(x)\left[T_{k i}(s, x)\right]=M_{k p}(s, x)
\end{aligned}
$$

It then follows that

$$
\begin{aligned}
& \int_{B}\left[L_{k p}(s, x) t_{k}(s)-M_{k p}(s, x) u_{k}(s)\right] d B(s)=t_{p}(x), \quad \text { if } x \in D \ldots \\
& \int_{B}\left[L_{k p}(s, x) t_{k}(s)-M_{k p}(s, x) u_{k}(s)\right] d B(s)=0, \quad \text { if } x \in D \ldots \ldots
\end{aligned}
$$

Eqs. $11 a$ and $14 a$ are the dual equations (Buecker 1973) for any point $x$ in the domain.

\section{Weighted Residual Method}

Consider the problem, as in Fig. 1, with the governing equation: 


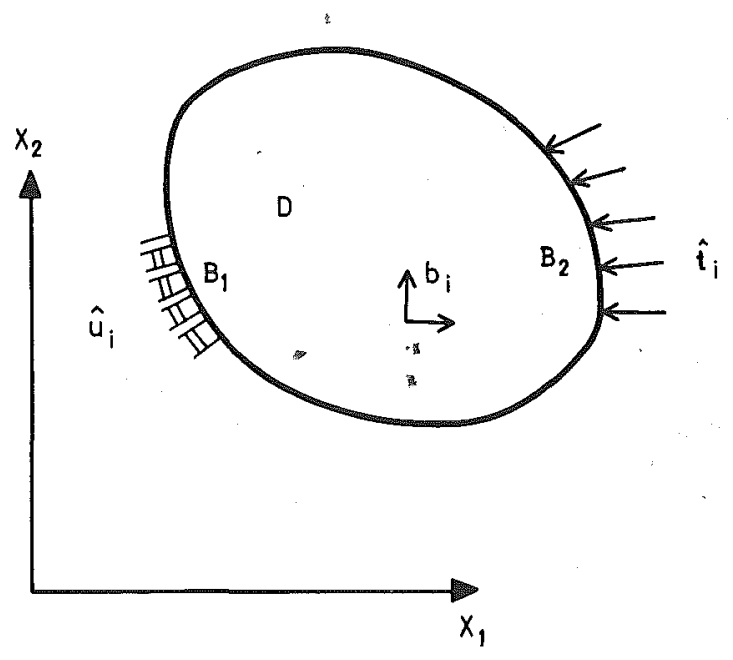

FIG. 1. Classical Elasticity Problem

$D_{i j}(x) u_{j}(x)=0, \quad$ if $x \in D$

and the boundary conditions:

$u_{i}(x)=\hat{u}_{i}(x), \quad$ if $x$ on $B_{1}$

$t_{i}(x)=\hat{t}_{i}(x), \quad$ if $x$ on $B_{2}$

where $D_{i j}$ is defined in Eq. $4 ;\left(^{\wedge}\right)=$ a prescribed value at the boundary; and $B=B_{1}+B_{2}$.

In solving the given boundary value problem, the vanishing right-hand side terms are practically not zero and have some errors or residuals $R_{1}$, $R_{2}$, and $R_{3}$. In the weighted residual method, the residuals are minimized with respect to preselected weighting functions $W_{1}, W_{2}$, and $W_{3}$, respectively, as in the following manner:

$$
\begin{aligned}
& \int_{D} W_{1 i}(x) D_{i j} u_{j}(x) d V(x)=\int_{B_{1}} W_{2 i}(x)\left[u_{i}(x)-\hat{u}_{i}(x)\right] d B(x) \\
& -\int_{B_{2}} W_{3 i}(x)\left[t_{i}(x)-\hat{t}_{i}(x)\right] d B(x) \ldots \ldots \ldots \ldots \ldots \ldots
\end{aligned}
$$

This statement of minimizing weighted residuals is a very general one (Brebbia et al. 1984); we shall see immediately that Eqs. $11 a$ and $14 a$ are merely special cases of Eq. 17. Setting $v_{i}=W_{1 i}$ and $v_{j}=W_{1 j}$ in Eq. 6, the right-hand side of Eq. 17 becomes

$$
\int_{D} W_{1 i} D_{i j} u_{j} d V=\int_{D} u_{i} D_{i j} W_{1 j} d V+\int_{B} W_{1 i} B_{i j} u_{j} d B-\int_{B} u_{i} B_{i j} W_{i j} d B
$$




$$
\begin{aligned}
& =\int_{D} u_{i} D_{i j} W_{1 j} d V+\int_{B_{1}} W_{1 i} t_{i} d B+\int_{B_{2}} W_{1 i} \hat{t}_{i} d B \\
& -\int_{B_{1}} \hat{u}_{i} B_{i j} W_{1 j}(x) d B(x)-\int_{B_{2}} u_{i} B_{i j} W_{1 j} d B \ldots \ldots
\end{aligned}
$$

upon taking the boundary conditions (Eqs. 16) into account. Substituting Eq. 18 into Eq. 17 and considering Eqs. $5 a, 7,8$, and 15 and that

$$
\begin{aligned}
& W_{1 i}(x)=W_{3 i}(x)=U_{i k}(x, s) e_{k}(s) \ldots \ldots \ldots \\
& D_{i j}(x) W_{1 j}(x)=D_{i j}(x) U_{j k}(x, s) e_{k}(s)=-\delta_{i k}(x, s) e_{k}(s) \ldots \ldots \\
& B_{i j}(x) W_{1 j}(x)=W_{2 i}(x)=B_{i j}(x) U_{j k}(x, s) e_{k}(s)=T_{i k}(x, s) e_{k}(s)
\end{aligned}
$$

Since $e_{k}(s)$ is a unit-concentrated load at point $s$, we cancel the same terms and finally have

$$
\int_{B} U_{i k}(x, s) t_{i}(x)-T_{i k}(x, s) u_{i}(x) d B(x)=u_{k}(s) \ldots \ldots \ldots \ldots \ldots
$$

which is exactly the same Somigliana identity as given in Eq. $10 a$.

On the other hand, upon choosing another set of weighting functions

$$
\begin{aligned}
& \underline{W}_{1 i}=L_{i p}(x, s) e_{p}^{*}(s) \\
& \underline{W_{2 i}}=M_{i p}(x, s) e_{p}^{*}(s) \\
& \underline{W_{3 i}}=L_{i p}(x, s) e_{p}^{*}(s)
\end{aligned}
$$

we have

$$
\begin{aligned}
& \int_{D} \underline{W}_{1 i}(x) D_{i j} u_{j} d V(x)=\int_{D} u_{i}(x) D_{i j} \underline{W}_{1 j}(x) d V(x) \\
& +\int_{B} \underline{W}_{1 i} B_{i j} u_{j}(x) d B(x)-\int_{B} u_{i}(x) B_{i j} \underline{W}_{1 j}(x) d B(x) \\
& =\int_{B_{1}}\left[u_{i}(x)-\hat{u}_{i}(x)\right] \underline{W_{2 i}}(x) d B(x)-\int_{B_{2}}\left[\hat{t}_{i}(x)-t_{i}(x)\right] \underline{W_{3 i}}(x) d B(x) \ldots
\end{aligned}
$$

which due to Eqs. 12 and 13 becothes

$$
\begin{aligned}
& B_{p i}(s)\left[\int_{D} W_{1 i}(x) D_{i j} u_{j} d V(x)\right]=B_{p j}(s)\left[\int_{D} u_{i}(x) D_{i j} W_{1 j}(x) d V(x)\right. \\
& \left.+\int_{B} W_{1 i} B_{i j} u_{j}(x) d B(x)-\int_{B} u_{i}(x) B_{i j} W_{1 j}(x) d B(x)\right]
\end{aligned}
$$


TABLE 1. Meanings of Fundamental Solutions; $(j)=$ in $j$-direction

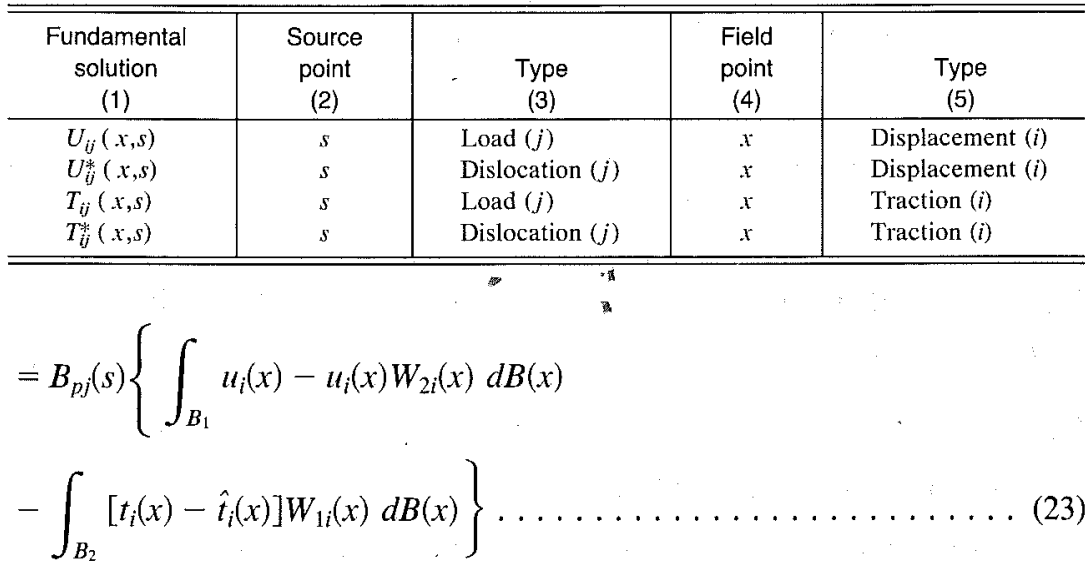

In this way there emerges the equation:

$$
\int_{B}\left[L_{i p}(x, s) t_{i}(x)-M_{i p}(x, s) u_{i}(x)\right] d B(x)=t_{p}(s) \ldots \ldots \ldots \ldots \ldots
$$

Thus we obtain Eq. 14a again.

Before leaving this section, we note that $\mathrm{Eq} .17$ is of value not only in its generality, but also because it can serve as a bridge for a coupled use of the BEM and the finite-element method (Brebbia 1984).

\section{By Physical Meaning}

The method to be presented is based on the superposition principle due to the linear operator theory and can be understood easily in physical sense. It may be cataloged under the theory of potential. In BEM terminology, it is frequently called the indirect method. First, we determine four free-space Green's functions or fundamental solutions as shown in Table 1. Then invoking the superposition principle, we have

$$
\begin{aligned}
& u_{i}(x)=\int_{B} U_{i k}(x, s) \phi_{k}(s) d B(s)+\int_{B} U_{i k}^{*}(x, s) \psi_{k}(s) d B(s) \ldots \ldots \ldots \\
& t_{i}(x)=\int_{B} T_{i k}(x, s) \phi_{k}(s) d B(s)+\int_{B} T_{i k}^{*}(x, s) \psi_{k}(s) d B(s) \ldots \ldots \ldots
\end{aligned}
$$

Here the load $\phi_{k}(s)$ on the boundary $B$ must be understood to be the relative traction, i.e., the difference between the traction applied on the boundary to the domain $D_{i}$ under consideration and that applied to the exterior $D_{e}$, which is a complement to the considered domain. If the traction applied to the exterior is assumed to vanish, as shown later in the right-hand side of Eq. $27 b$, the load $\phi_{k}(s)$ of Eq. 20 becomes the traction $t_{k}(s)$ applied to the domain considered. Similarly, in Eqs. 25 and 26, the dislocation $\psi_{k}(s)$ on the boundary is synonymous with the relative displacement and can be interpreted to be the displacement $u_{k}(s)$ of the point $s$ of 
the bounding boundary of the domain that is being considered if the displacement of the boundary bounding the exterior is taken to be zero, as will be shown in the right-hand side of Eq. $28 b$. Thus

$$
\begin{aligned}
& \int_{B} U_{i k}(x, s) t_{k}(s) d B(s)+\int_{B} U_{i k}^{u}(x, s) u_{k}(s) d B(s)=u_{i}(x), \quad \text { if } x \in D_{i}(27 a) \\
& \int_{B} U_{i k}(x, s) t_{k}(s) d B(s)+\int_{B} U_{i k}^{*}(x, s) u_{k}(s) d B(s)=0, \quad \text { if } x \in D_{e} \ldots(27 b) \\
& \int_{B} T_{i k}(x, s) t_{k}(s) d B(s)+\int_{B} T_{i k}^{*}(x, s) u_{k}(s) d B(s)=t_{i}(x), \quad \text { if } x \in D_{i} .(28 a) \\
& \int_{B} T_{i k}(x, s) t_{k}(s) d B(s)+\int_{B} T_{i k}^{*}(x, s) u_{k}(s) d B(s)=0, \quad \text { if } x \in D_{e} \ldots(28 b)
\end{aligned}
$$

We note that the kernel functions of Eqs. 11, 14, 25, and 26 appear to be different in the orders of subscripts and arguments; however, we shall soon see in the following section, where we will prove four important theorems, that they are equivalent in essence.

\section{Four Lemmas and Consistency of Derivations}

To show the consistencies of Eqs. 11 and 25 and of Eqs. 14 and 26, which are derived from different approaches, we must prove the following four lemmas:

Lemma (a): $\quad U_{i k}(x, s)=U_{k i}(s, x)$

Lemma (b): $\quad U_{i k}^{*}(x, s)=-T_{k i}(s, x)$

Lemma (c): $\quad T_{i k}(x, s)=L_{k i}(s, x)$

Lemma (d): $T_{i k}^{*}(x, s)=-M_{k i}(s, x)$

Lemmas (a) and (b) can be proved using Betti's law. Lemma (a) is well known in literature and its proof is skipped herein.

To prove lemma (b), we divide the infinite domain by an imaginary boundary $B$ into $D^{+}$and $D^{-}$. Let $s \in D_{i}$; then both Eq. $11 a$ and Eq. $11 b$ still hold, reading

$$
\begin{aligned}
& \int_{B}\left[U_{k i}(s, x) t_{k}^{+}(s)-T_{k i}(s, x) u_{k}^{+}(s)\right] d B(s)=u_{i}(x) \ldots \ldots \ldots \ldots \\
& \int_{B}\left[U_{k i}(s, x) t_{k}^{-}(s)+T_{k i}(s, x) u_{k}^{-}(s)\right] d B(s)=0 \ldots \ldots \ldots \ldots
\end{aligned}
$$

where $T_{k i}(s, x)$ is associated with $n^{+}(s)$. Addition of Eqs. $30 a$ and $30 b$ gives

$\int_{B}\left\{U_{k i}(s, x)\left[t_{k}^{+}(s)+t_{k}^{-}(s)\right]-T_{k i}(s, x)\left[u_{k}^{+}(s)-u_{k}^{-}(s)\right]\right\} d B(s)=u_{i}(x)$ 
If $u_{k}^{+}(s)-u_{k}^{-}(s)=\delta_{k j}\left(s-x^{\prime}\right)$ and $t_{k}^{+}(s)+t_{k}^{-}(s)=0$, i.e., if a dislocation source in the $j$-direction is applied at $x^{\prime}$, then $u_{i}(x)=U_{i j}^{*}\left(x, x^{\prime}\right)$ by definition, and thus Eq. 31 becomes

$-T_{i j}\left(x^{\prime}, x\right)=U_{i j}^{*}\left(x, x^{\prime}\right)$

which is identical to lemma (b).

After the proof of lemmas (a) and (b), we are in a position to prove lemmas (c) and (d). By definition of Eq. 12 and using lemma (a), we have $L_{k p}(s, x)=B_{p i}(x)\left[U_{k i}(s, x)\right]$

$L_{k p}(s, x)=B_{p i}(x)\left[U_{i k}(x, s)\right]$

$L_{k p}(s, x)=T_{p k}(x, s)$

Similarly, by definition of Eq. 13 and lemma (b)

$M_{k p}(s, x)=B_{p i}(x)\left[T_{k i}(s, x)\right]$

$M_{k p}(s, x)=B_{p i}(x)\left[-U_{i k}(x, s)\right]$

$M_{k p}(s, x)=-T_{p k}^{*}(x, s)$

This completes the proof of the four lemmas.

It is worthy noting that, in real calculations, only three kernels need to be determined because of lemma (c). We also note that from the preceding it can be shown that

$$
\begin{aligned}
& B_{p i}(x)\left[U_{i k}(x, s)\right]=T_{p k}(x, s) \ldots \\
& B_{p k}(s)\left[U_{i k}(x, s)\right]=-U_{i p}^{*}(x, s)
\end{aligned}
$$

This subtle result cautions us that the traction derivative of an influence function with respect to the coordinate of the field point $x$ does indeed represent another influence function, which describes the state of a different response due to the same sigularity source. On the contrary, the traction derivative of an influence function with respect to a source point coordinate is another influence function of the same response as the original influence function but due to a different sigularity source (Heise 1978).

\section{Derivations of Dual Boundary Integral. Equations}

In this section we derive boundary integral equations for problems of elastic media with or without cracks. For the integral equations derived in the previous sections to be useful, it is very important for us to be able to move the point for which the equations are written to the boundary. Since in doing so, the resulting equations involve boundary unknowns, this makes one less dimension to the problem and thus greatly lowers the demand on computation time and storage space. This desirable feature is not at all trivial for solving real problems and deserves intensive study. As we shall see later, the concepts of principal values and the trace operator of extension help contribute to the success achieved herein. For clarity, we will retain a few duplications that have already been presented in the derivation of the domain equations. 


\section{Direct Method}

By direct method, we are referring to methods based on a relationship of reciprocality (for the elasticity examined here, Betti's law) or a variational approach such as the weighted residual method already discussed. There the domain integral equations, Eqs. 11 and 14, are good for a point on the interior or exterior of the domain. When we move the point to the boundary, we are immediately confronted with the problem of singularities and improper integrals.

It is well known that Eq. $11 a$, where $x \in D_{i}$, thus becomes

$$
\int_{B} U_{k i}(s, x) t_{k}(s) d B(s)+\beta_{i j} u_{j}(x)-\mathrm{CPV} \int_{B} T_{k i}(s, x) u_{k}(s) d B(s)=\delta_{i j} u_{j}(x) \ldots \ldots
$$

where $x \in B$, and Eq. $11 b$ where $x \in D_{e}$ becomes

$$
\int_{B} U_{k i}(s, x) t_{k}(s) d B(s)+\left(-\delta_{i j}+\beta_{i j}\right) u_{j}(x)-\mathrm{CPV} \int_{B} T_{k i}(s, x) u_{k}(s) d B(s)=0 \ldots
$$

where $x \in B . \beta_{i j}(x)$ depends on the solid angle and on the configuration of the corner at $x$ of the boundary and on Poisson's ratio of the material of the body $D$. At a smooth boundary, $\beta_{i j}$ reduces to $1 / 2 \delta_{i j}$. Hartmann (1981, 1982) contains a detailed discussion on the subject. We note that no matter where $x$ comes from, the resulting equations, Eqs. 36 and 37, are identical. Setting $c_{i j}=\delta_{i j}-\beta_{i j}$, Eqs. 36 and 37 become

$c_{i j} u_{j}(x)=\int_{B} U_{k i}(s, x) t_{k}(s) d B(s)-\mathrm{CPV} \int_{B} T_{k i}(s, x) u_{k}(s) d B(s) \ldots \ldots$

where the point $x$ is located on the boundary, and CPV $=$ the Cauchy principal value. Now applying the traction operator to Eq. 38, and noting that

$$
\begin{aligned}
& B_{p i}(x) \int_{B} U_{k i}(s, x) t_{k}(s) d B(s)=B_{p i}(x) \int_{B-B_{\mathrm{E}}} U_{k i}(s, x) t_{k}(s) d B(s) \\
& =\mathrm{CPV} \int_{B} L_{k p}(s, x) t_{k}(s) d B(s) \ldots \ldots \ldots \ldots \ldots \ldots \ldots \ldots \ldots \ldots \ldots \ldots \ldots \ldots \ldots \ldots \ldots \ldots
\end{aligned}
$$

where the first equality results from the integral over the small detour at $x \in B_{\varepsilon}$, where $B_{\varepsilon}=$ a small spherical or circular detour of radius $\varepsilon$ and center at $x$, vanishing, and the second equality stems from the boundary terms due to the traction operation using Leibnitz' rule canceling themselves out, and defining that

$B_{p i}(x)\left[\mathrm{CPV} \int_{B} T_{k i}(s, x) u_{k}(s) d B(s)\right] \equiv \mathrm{HPV} \int_{B} M_{k p}(s, x) u_{k}(s) d B(s)$

we have

$c_{p j} t_{j}(x)=\mathrm{CPV} \int_{B} L_{k p}(s, x) t_{k}(s) d B(s)-\mathrm{HPV} \cdot \int_{B} M_{k p}(s, x) u_{k}(s) d B(s)$ 
where $x$ is on the boundary; and HPV = the Hadamard principal value (Hadamard 1952). Eqs. 38 and 41 are the dual boundary integral equations for any boundary point.

\section{Indirect Method (Potential Theory)}

For convenience we define

$$
\begin{aligned}
& \psi_{i}^{-}(x)=\lim _{y \rightarrow x} u_{i}(y) \\
& U_{i}^{-}(x)=\lim _{y \rightarrow x} t_{i}(y) \\
& \psi_{i}^{+}(x)=\lim _{z \rightarrow x} u_{i}(z) . \\
& U_{i}^{+}(x)=\lim _{z \rightarrow x} t_{i}(z) .
\end{aligned}
$$

in which $x \in B, y \in D_{i}$, and $z \in D_{e}$. Now applying the trace operator to Eq. 25 by taking the points of the interior and exterior of the boundary, we have

$$
\begin{aligned}
& \psi_{i}^{-}=\int_{B} U_{i k}(x, s) \phi_{k}(s) d B(s)+\beta_{i j} \psi_{j}(x)+\mathrm{CPV} \int_{B} U_{i k}^{*}(x, s) \psi_{k}(s) d B(s) \ldots \ldots \\
& \psi^{+}=\int_{B} U_{i k}(x, s) \phi_{k}(s) d B(s)+\left[-\delta_{i j}+\beta_{i j}\right] \psi_{j}(x)+\mathrm{CPV} \int_{B} U_{\breve{k}}^{*}(x, s) \psi_{k}(s) d B(s)
\end{aligned}
$$

Application of the traction operator to Eqs. 43 and 44 gives

$$
\begin{aligned}
& U_{i}^{-}=\beta_{i j} \phi_{j}+\operatorname{CPV} \int_{B} T_{i k}(x, s) \phi_{k}(s) d B(s)+\operatorname{HPV} \int_{B} T_{i k}^{*}(x, s) \psi_{k}(s) d B(s) . \\
& U_{i}^{+}=\operatorname{CPV} \int_{B} T_{i k}(x, s) \phi_{k}(s) d B(s)+\left[-\delta_{i j}+\beta_{i j}\right] \phi_{j}(x)+\operatorname{HPV} \int_{B} T_{i k}^{*}(x, s) \psi_{k}(s) d B(s)
\end{aligned}
$$

The reasons for the presence of $\beta_{i j}$, the Cauchy principal values, and the Hadamard principal values are similar to those elaborated previously. Note that Eqs. 43-46 of the indirect method are very general, involving the single-layer and double-layer potentials and their traction derivatives.

\section{Equivalence of Direct and Indirect Methods}

The equivalence of the direct and indirect methods can be observed by noting the four lemmas and arranging a few equalities. To show the equivalence of Eqs. 11 and Eqs. 43 and 44, we invoke lemmas (a) and (b) and require that $\phi_{i}=t_{i} ; \psi_{i}=u_{i} ; \Psi_{i}^{-}=u_{i} ;$ and $\Psi^{+}=0$. Thus we obtain Eq. 38. Similarly, to show the equivalence of Eqs. 14 and Eqs. 45 and 46; we recall lemmas (c) and (d) and require that $\phi_{i}=t_{i} ; \psi_{i}=u_{i} ; U_{i}^{-}=t_{i} ;$ and $U_{i}^{+}$ $=0$. Thus we obtain Eq. 41 .

From the equivalence of Eqs. 11 and Eqs. 43 and 44, and Eqs. 14 and Eqs. 45 and 46, it is obvious that the direct and indirect methods are of 


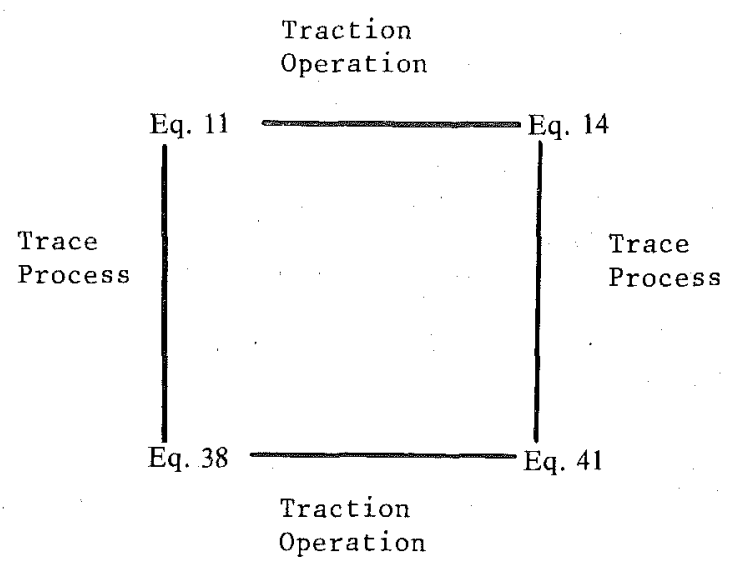

FIG. 2. Commutative Diagram

little fundamental difference in the sense of the dual equations (Heise 1978).

\section{Commutativity}

It is interesting to discover that Eq. 41 can also be derived by directly applying the trace operator of extension to Eq. 14. Thus

$c_{i j} t_{j}(x)=\mathrm{CPV} \int_{B} L_{k i}(s, x) t_{k}(s) d B(s)-\lim _{y \rightarrow x} \int_{B} M_{k i}(s, y) u_{k}(s) d B(s) \ldots \ldots \ldots \ldots$

Comparison between Eqs. 41 and 47 reveals

$$
\begin{aligned}
& \operatorname{HPV} \int_{B} M_{k p}(s, x) u_{k}(s) d B(s)=B_{p i}(x)\left[\mathrm{CPV} \int_{B} T_{k i}(s, x) u_{k}(s) d B(s)\right] \\
& =\lim _{y \rightarrow x} \int_{B} M_{k p}(s, y) u_{k}(s) d B(s)=\mathrm{HPV} \int_{B} \lim M_{k p}(s, y) u_{k}(s) d B(s)
\end{aligned}
$$

using Eq. 40 and due to the uniqueness of the solution.

Remember that in deriving Eq. 41, the trace operation of extension is taken first and then the traction operation, while in arriving at Eq. 47, the order of the two operations is reversed. But Eq. 48 confirms that the two equations are equal; thus the commutativity of the trace and traction operators is established. To illustrante the idea further, a commutative diagram is shown in Fig. 2, in which we have two paths to Eq. 41. When we apply the traction operator to Eq. 38 with respect to $x$ from path 1 , it must be noted that the upper and lower bounds of the Cauchy integral is a function of $x$; the Leibnitz rule must be used, and finiteness is proved logically. In the other path (path 2), the existence of the traction derivative of the double-layer potential is well known in the literature of applied mathematics (Kupradze 1979). 


\section{Analytical and Numerical Examples}

In this section we present one closed-form solution and one anlaytical solution to show the validity of the theory.

Consider a straight-line crack in the two-dimensional infinite domain subjected to an opening-up pressure $P$ uniformly exerted on the surfaces of the crack extending from $x=-1$ to $x=1$. The formulation of the problem is reduced from our dual integral equations to a Fredholm equation

$$
\frac{1}{2} \Delta t_{2}(x)=-\sigma_{22}(x)=P=\mathrm{HPV} \int_{-1}^{\mathrm{T}} \frac{-\stackrel{\leftrightarrow}{G}}{(1-v) \pi} \frac{u_{2}(s)}{(x-s)^{2}} d s \ldots \ldots \ldots
$$

by using the symmetry conditions, $\Delta u_{1}=0$; and $\Delta u_{2}=2 u_{2}$.

First, we derive the exact solution by series expansion, assuming

$$
u_{2}(s)=\sum_{i=0}^{\infty} a_{i} U_{i}(s) \sqrt{1-s^{2}}
$$

where $U_{i}(s)=$ the Chebyshev polynomials of the second kind; and $a_{i}=$ constants to be determined. Substituting Eq. 50 into Eq. 49

$$
-P=\frac{G}{(1-\nu) \pi} \sum_{i=0}^{\infty} \operatorname{HPV} \int_{-1}^{1} a_{i} U_{i}(s) \frac{\sqrt{1-s^{2}}}{(x-s)^{2}} d s
$$

According to Hashmi and Delves (1982)

$$
\operatorname{CPV} \int_{-1}^{1} \frac{U_{i}(s) \sqrt{1-s^{2}}}{(s-x)} d s=-\pi T_{i+1}(x) \ldots \ldots \ldots \ldots \ldots
$$

where $T_{i+1}(x)=$ the Chebyshev polynomials of the first kind. Using Hadamard's definition and the property of the Chebyshev polynomials

$$
\begin{aligned}
& \operatorname{HPV} \int_{-1}^{1} \frac{U_{i}(s) \sqrt{1-s^{2}}}{(s-x)^{2}} d s \\
& =\frac{d}{d x}\left[\operatorname{CPV} \int_{-1}^{1} \frac{U_{i}(s) \sqrt{1-s^{2}}}{(s-x)} d s\right]=-\pi(i+1) U_{i}(x) \ldots \ldots \ldots
\end{aligned}
$$

Thus

$$
\frac{P(1-\nu)}{G}=\sum_{i=0}^{\infty} a_{i}(i+1) U_{i}(x) \ldots \ldots \ldots \ldots \ldots \ldots \ldots \ldots \ldots \ldots
$$

which gives

$$
\begin{aligned}
& a_{i}=\frac{P(1-v)}{G}, \quad \text { if } i=0 \\
& a_{i}=0, \quad \text { if } i \neq 0 \ldots \ldots
\end{aligned}
$$



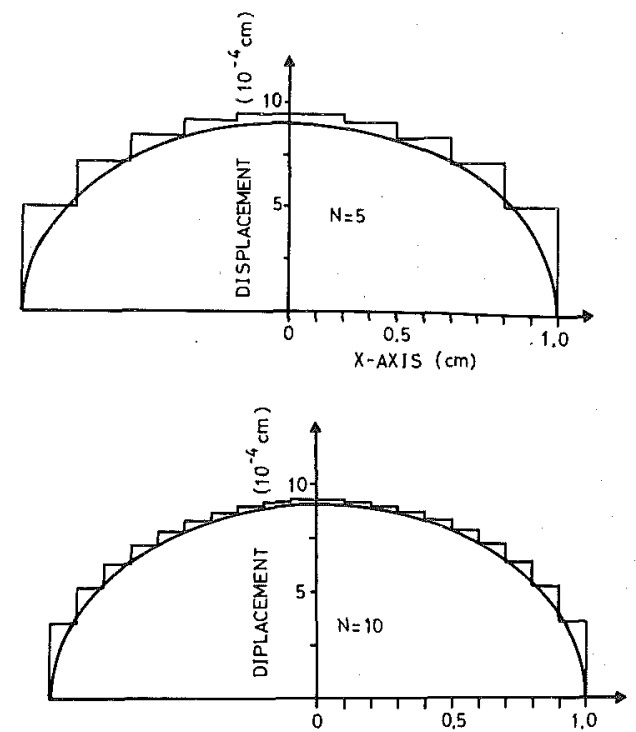

$X-A X I S(\mathrm{~cm})$

管

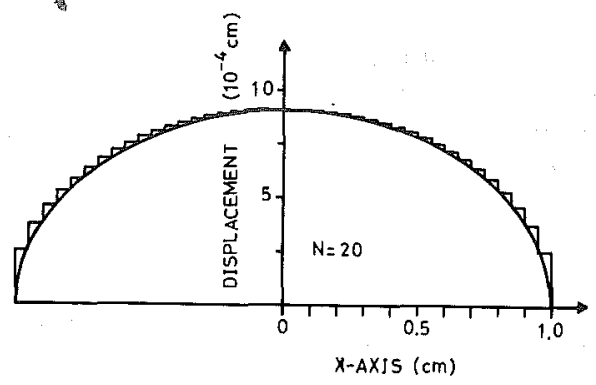

FIG. 3. Crack Profile of Numerical and Analytical Solutions

The exact solution is

$u_{2}(s)=\frac{P(1-v)}{G} \sqrt{1-s^{2}}$

In numerical analysis, choosing constant elements of length $2 l$ for interpolating $u_{2}$ of Eq. 49 , we carry out the integration of the integrand containing $M_{22}(s, x)$ as detailed in the following.

Using a constant element scheme, we find that the integration for a singular element $|x|<l$ is

$$
\begin{aligned}
& \text { HPV } \int_{-l}^{l} \frac{1}{(x-s)^{2}} d s=\lim _{\varepsilon \rightarrow 0}-\frac{2}{\varepsilon}+\left.\frac{1}{x-s}\right|_{s=-1} ^{s=x-\varepsilon}+\left.\frac{1}{x-s}\right|_{s=x+\varepsilon} ^{s=l} \\
& =\lim _{\varepsilon \rightarrow 0}-\frac{2}{\varepsilon}+\frac{2}{\varepsilon}-\frac{1}{x+l}+\frac{1}{x-l}=\frac{2 l}{x^{2}-l^{2}} \ldots \ldots \ldots \ldots \ldots \ldots
\end{aligned}
$$


For a regular element $|x|>l$, the integration is

$$
\int_{-l}^{l} \frac{1}{(x-s)^{2}} d s=\left.\frac{1}{x-s}\right|_{s=-1} ^{s=1}=\frac{2 l}{x^{2}-l^{2}} \ldots \ldots \ldots \ldots \ldots \ldots \ldots
$$

which is identical to the results for a singular element. Crouch (1983) found no difference in the results when basing them on the Papkovitch-Neuber potential function. The numerical result is shown in Fig. 3.

\section{Conclusion}

In this paper, we have presented dual integral equations for solving general linear elasticity problems of finite or infinite domain with degenerate boundaries such as cracks, cusps, corners, and holes of any irregular shape. The equations can be viewed as a pair of fundamental formulations of the theory of elasticity and linear-elastic fracture mechanics. The discovery of the equations is also important from the point of view of applied mathematics.

The main motivation for developing the dual boundary integral equations comes from their use in solving crack problems. Crack problems cannot be solved without the introduction of the kernel $T_{i j}^{*}$ (or $M_{j i}$ ) or equivalent forms. The absence of $T_{i j}^{*}$ is why Somigliana's identity cannot solve the crack problems.

The derivation is logical; it leads naturally to the concepts of Cauchy and Hadamard principal values. Thus, the singularity often present in problems of this kind presents no particular difficulties; instead, it makes the problem much more well-posed and therefore accelerates the speed of solution.

The derivation is rigorous in that it is completed from six routes, and the results of each of the six have been proved equivalent. The six routes consist of three approaches, Betti's law, the weighted residual method, and the theory of potential, and of two operations, traction and trace, in different orders of application.

The possible applications of the concept of dual integral equations are obviously not confined to the fields of elasticity and fracture mechanics. Other areas, e.g., potential flow around cutoff walls, acoustic waves impinging on screen, airfoil dynamics, and magnetic wave across antenna, have the problem of geometry degeneracy and can be solved using appropriate dual integral equations.

\section{Appendix I. References}

Banerjee, P. K., and Butterfield, R. (1981). Boundary element method in engineering science. McGraw-Hill, New York, N.Y.

Brebbia, C. A., Tells, J. C. F., and Wrobel, L. C. (1984). Boundary element techniques. Springer-Verlag.

Buecker, H. F. (1973). "Field singularities and related integral representations." Mechanics of fracture, Vol. 1, G. C. Sih, ed.

Crouch, S. L., and Starfield, A. M. (1983). "Boundary element methods in solid mechanics." Berne Convention.

Cruse, T. A. (1975). "Computational applications in applied mechanics." $A M D-11$, T. A. Cruse and Rizzo, eds. ASME, 31-46.

Elliott, D. (1980). "Ninety years on." Application and numerical solution of 
integral equations, R. S. Anderson, F. R. de Hoog, and M. A. Lukas, eds. Noordoff.

Hadamard, J. (1952). Lectures on Cauchy's problem in linear partial differential equations, Dover Publications, Inc., Mineola, N.Y.

Hartmann, F. (1981). "The Somigliana identity on piecewise smooth surfaces." $J$. Elast., 11, 403-423.

Hartmann, F. (1982). "Elastic potentials on piecewise smooth surfaces." J. Elast., $12,31-50$.

Hashmi, S. H., and Delves, L. M. (1982). "The fast Galerkin method for the solution of Cauchy singular integral equations." in Treatment of integral equations by numerical methods, Baker et al., eds., Academic Press, Orlando, Fla.

Heise, U. (1978). "Application of the singularity method for the formulation of plane elastostatical boundary value problems as integral equations, "Acta Mech., $31,33-69$.

Kupradze, V. D. (1963). Potential methods in the theory of elasticity. translated from Russian by Israel Program for Scientific Translation, Jerusalem, Israel.

Kupradze, V. D., et al. (1979). Three-dimensional problems of the mathematical theory of elasticity and thermoelasticity. North-Holland Publ. Co.

Lardner, R. W. (1972). "Dislocation layers and boundary value problems of plane elasticity." Q.J. Mech. Math. 25, 45-61.

Lauricella, G. (1909). "Sur Pintégration de Péquation relative à Pequilibre des plaques élastiques encastrées." Acta Math., 32 (in French).

Ligget, J. A., and Liu, P. L-F. (1983). The boundary integral equation method for porous media flow. Allen \& Unwin.

Massonnet, C. E. (1965). "Numerical use of integral procedures." Stress analysis, O. C. Zienkiewicz and G. S. Holister, eds., John Wiley and Sons, London, U.K.

Mikhlin, S. G. (1970). Mathematical physics: an advanced course. Elsevier.

Muskhelishvili, N. I. (1953). Some basic problems of the mathematical theory of elasticity. Noordoff.

Rizzo, F. J. (1967). "An integral equation approach to boundary value problems of classical elastostatics." Q. Appl. Math., 40, 83-95.

\section{APPENDIX II. NotATION}

The following symbols are used in this paper:

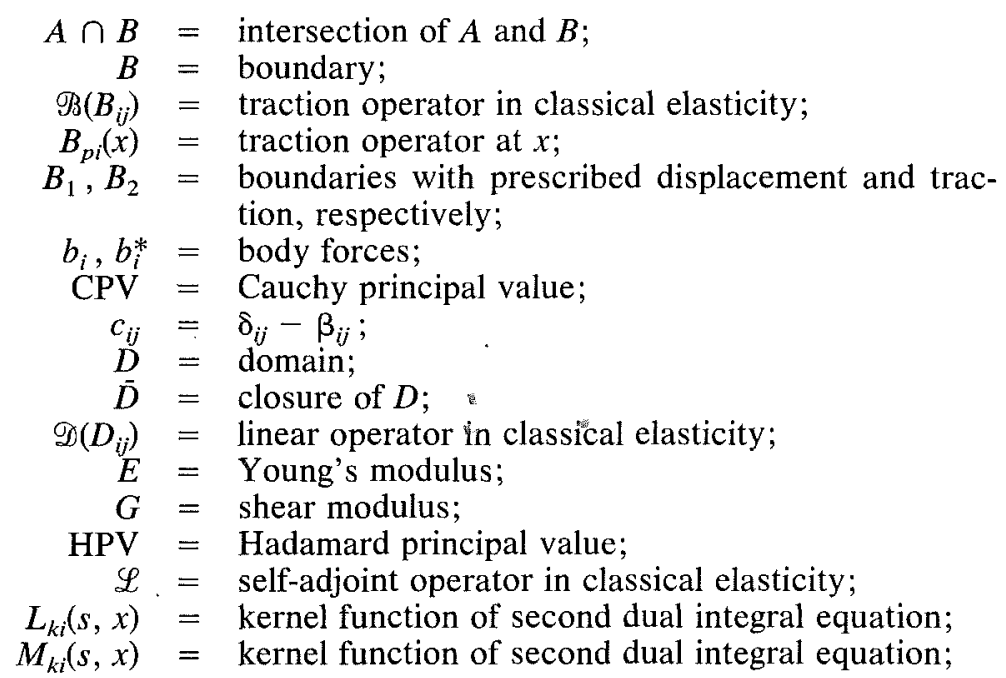




$$
\begin{aligned}
n_{i} & =\text { normal vector; } \\
p & =\text { pressure at crack surface; } \\
R^{n} & =\text { considered } n \text {-dimensional domain; } \\
R_{1}, R_{2}, R_{3} & =\text { residuals; } \\
S^{+}, S^{-} & =\text {boundary near boundary } S \\
S_{\infty} & =\text { infinite boundary; } \\
s \in D & =s \text { is element of } D ; \\
s \in D & =s \text { is not element of } D ; \\
T_{i j}(x, s) & =i \text {-component traction at } x \text { due to concentrated } j \text { - } \\
T_{i j}^{*}(x, s) & =\text { direction load at } s ; \\
t_{i}, t_{i}^{*} & =\text { component traction at } x \text { due to } j \text {-direction dislo- } \\
U_{i j}(x, s) & =i \text {-component displacement at } x \text { due to concentrated } \\
U_{i j}^{*}(x, s) & =i \text {-direction load at } s ; \\
W_{2 i}, W_{3 i} & =\text { dislocation at } s ; \\
\beta_{i j} & =\text { weighting functions; } \\
\delta_{i j} & =\text { Kronecker delta; } \\
\delta_{i j}(x, s) & =\text { concentrated source at } s \text { in } j \text {-direction; } \\
\lambda & =\text { Lame's constant; } \\
\nu & =\text { Poisson's ratio; } \\
\phi_{k} & =\text { single-layer density; } \\
\psi_{k} & =\text { double-layer density; and } \\
\partial_{i} & =\text { partial differentiation with respect to } i \text { th coordinate. } \\
&
\end{aligned}
$$

